\title{
Survival Analysis of Mobile Prepaid Customers Using the Weibull Distribution
}

\author{
Rafael R. Vargas \\ Deep Learning RD \\ info@deeplearningrd.com
}

September 2018

\begin{abstract}
This paper shows an approach to modeling Prepaid churn and customer survival in the Telecommunications Industry, specifically in emerging markets where Prepaid subscriptions are the norm. We do so by applying the Weibull family of negative exponential distributions. For the analysis, we have used the IBM Watson telco customer demo dataset ${ }^{1}$, a public dataset of churning customers. We show that the Weibull distribution is a better fit for modeling Prepaid scenarios that display a high churn onset at the beginning but flattens out as time $t$ progresses. We then contrast our model with traditional methods of churn estimation and compare results between the different approaches.
\end{abstract}

keywords churn, prepaid, survival analysis, mobile carriers, telecom

\section{Introduction}

In the Telecommunications Industry directly serving end-customers - i.e. Mobile carriers - subscribers are categorized into two segments: Prepaid, which have a pay-as-you-go rate plan without the formalities of a contract; and Postpaid, which are legally obliged by a contract to stay for an agreed amount of time using the company services, lest they be charged with penalties for not complying.

It follows from this that Prepaid customers are more prone to churn, that is to cancel their service subscription, than their postpaid counterparts. In developing markets such as Latin America and Africa, the ratio of Prepaid to Postpaid is $80: 20^{2}$, showing an idiosyncracy of favoring the perceived freedom of a no-contract offer, despite average price per minute being higher and handset subsidy being very low or non-existent ${ }^{3}$. The main reasons for this are a lower customer purchasing power and a consequent lack of attachment to contract obligations in general. Since a large portion of the companies' revenue 
is driven by Prepaid subscribers, two problems arise; in one hand, companies need to implement efficient Subscriber Retention Efforts (SRE) in order to keep Prepaid churn as low as possible; in the other hand, they need to minimize the payback time for all the Subscriber Acquisition Efforts (SAE) (handset subsidy, promotions and advertising costs) which could be affected due to early leakage in the base.

Since keeping Prepaid subscribers is of high importance for telecommunications companies, specially in the above mentioned geographic areas, they invest heavily in models that analyze customer behavior and predict churn in order to be proactive and take action before it actually happens.

\section{Churn Definitions}

A mobile carrier gains subscribers in any given period $t$ by activating contract lines also known as gross additions $(G A)$ to the base. In that same period, subscription cancellations or disconnections $(D C X)$ can occur. Total net additions $(N A)$ are then given by

$$
G A_{t}-D C X_{t}=N A_{t}
$$

which yields a subscriber base for period $t$. Churn is then defined as the ratio of $D C X$ over the average base:

$$
C_{t}=\frac{D C X_{t}}{\text { AvgBase }_{t}}
$$

In general, there are two flavors of churn, voluntary and involuntary. Voluntary churn refers to the customer directly terminating the subscription by contacting the company and canceling the service contract. Involuntary churn happens when the subscription ends following internal business rules which may vary from company to company.

It is a rule of thumb of the telecommunications industry to apply churn rules to Postpaid users after 60 days of no invoice payment and to Prepaid after 90 days of no recharging or topping-up the balance for making calls. We will focus on Prepaid, since there are already penalty clauses in place to balance out churn in Postpaid users and their predictability to cancel the service is, in some ways, more controlled and easier to foresee when compared to Prepaid subscribers. We will also focus on the involuntary type, which accounts for the vast majority of total Prepaid churn, because there is no contract to be voluntarily terminated. 


\section{Traditional Approach to Churn Modeling}

Historically, there have been methods for modeling churn based on customer history and the probability or likehood of a customers canceling their subscription at any given time. Following Figini $(2006)^{4}$, traditional churn models often rely on the following:

- An invariant factor, which accounts for the type of subscriber contract

- A dynamic factor that characterizes trend and company interaction with its subscribers

- A seasonality factor as a function of the subscribed service contract period

- Some variables accounting for external factor such as regulations and competition

Thus, the general traditional model could be given by:

$$
\mathrm{C}_{t}=\alpha(x, y)\left(1-\beta_{t-n}\right)+\gamma_{z}+\eta
$$

where $C_{t}$ is churn at time $t ; \alpha$ is the static factor given as a function of the type of contract, $x$ for Postpaid, $y$ for Prepaid; $\beta$ is the trend coefficient going back $n$ periods; $\gamma$ is a cycle factor accounting for a specific season $z$, as defined by the nature of the business and $\eta$ characterizes the external environment variables.

This model is good enough for mature markets where general regulations have been long implemented and competition is well established, so that there is no threat of disruption by a new player. Also, this model is more accurate to measure Postpaid churn due to its higher dependence on the time remaining to contract renewal. Nevertheless, it is not accurate enough to describe the more volatile Prepaid segment, which have a proclivity to present higher churn in the periods near subscriber activation rather than later in the customer tenure.

Furthermore, the traditional models assume a uniform distribution for the churn rate over time, but empirically the rate may follow a variety of distributions depending on the type of product and life cycle moment of the subscriber. Specially for the Prepaid segment, the choice of a uniform distribution tends to underestimate the churn rate in the initial stages of the subscription and to over-

estimate it in the maturity of the customer tenure. This is crucial for forecasting revenue and customer base, so it's necessary to have a more efficient method to estimate churn that reduces budget impact and maximize performance.

\section{Modeling churn as failure rate: a survival ap- proach}

Survival analysis deals with data where the outcome variable is the expected time until the occurrence of an event of interest, in our case churn. A Survival 
function gives the probability that a customer will stay active beyond a given time threshold. This is given by

$$
S(t)=P(\{T>t\})=\int_{t}^{\infty} f(u) d u=1-F(t),
$$

where $F(t)$ is the cumulative distribution function or CDF.

Churn, then, can be thought as a hazard function indicating the failure rate of staying active with the service subscription, i.e., the failure to survive after some give time time $t$. A hazard function is the ratio of the probability density function (PDF) $f(t)$ to the survival function $S(t)$, given by

$$
\frac{\frac{d F(t)}{d t}}{1-F(t)}=\frac{f(t)}{S(t)}
$$

Different hazard curves can exist depending on the type of churn or rather the moment where it is more prominent. The following diagram shows different phases of churn, employing the well known bathtub curve to model hazard over time.

Fig. 1. hazard rate curves

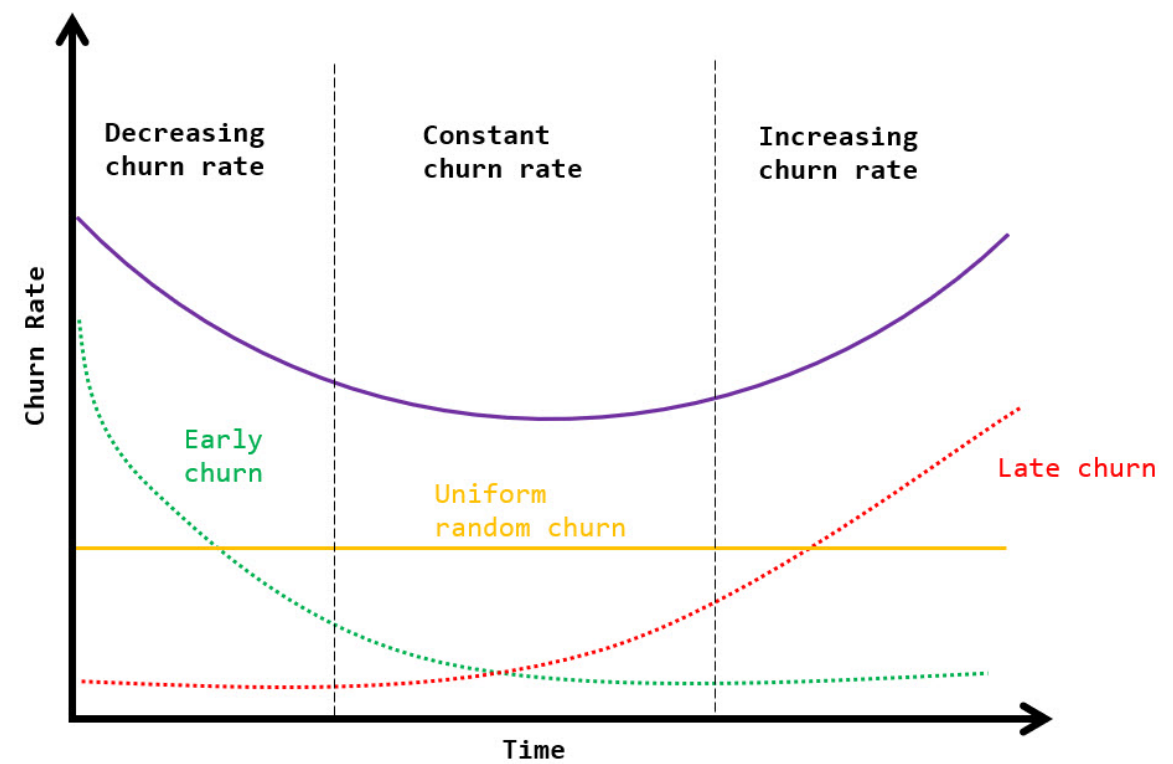

For estimating the survival curve, the Kaplan-Meier model is a good method if the number of case is representative and the survival times are known. The 
Kaplan-Meier estimator is given by:

$\widehat{S}(t)=\prod_{i: t_{i} \leq t}\left(1-\frac{d_{i}}{n_{i}}\right)$

with $t_{i}$ being the time when at least one event occurred, $d_{i}$ the number of events (i.e., contracts terminated) that happened at time $t_{i}$ and $n_{i}$ the contracts known to survive at time $t_{i}$.

A graph of the Kaplan-Meier estimator is a sequence of decreasing horizontal steps which, with a large enough sample size, approaches asymptotically the true survival function for the analyzed population. The value of the survival function between successive distinct sampled observations is assumed to be constant ${ }^{5}$.

\section{Data Analysis}

Following from the bathtub curve diagram, Prepaid customers fall in the Early churn rate area. This is congruent with the nature of the service, where customers have freedom to cancel at any time and often display a "try and buy" behavior. We have analyzed this assumptions against the IBM Watson telco customer demo dataset, which provides public churn data for Postpaid and Prepaid subscribers. The Kaplan-Meier estimator curves for the set are as follows:

Fig.2. Kaplan-Meier survival curves

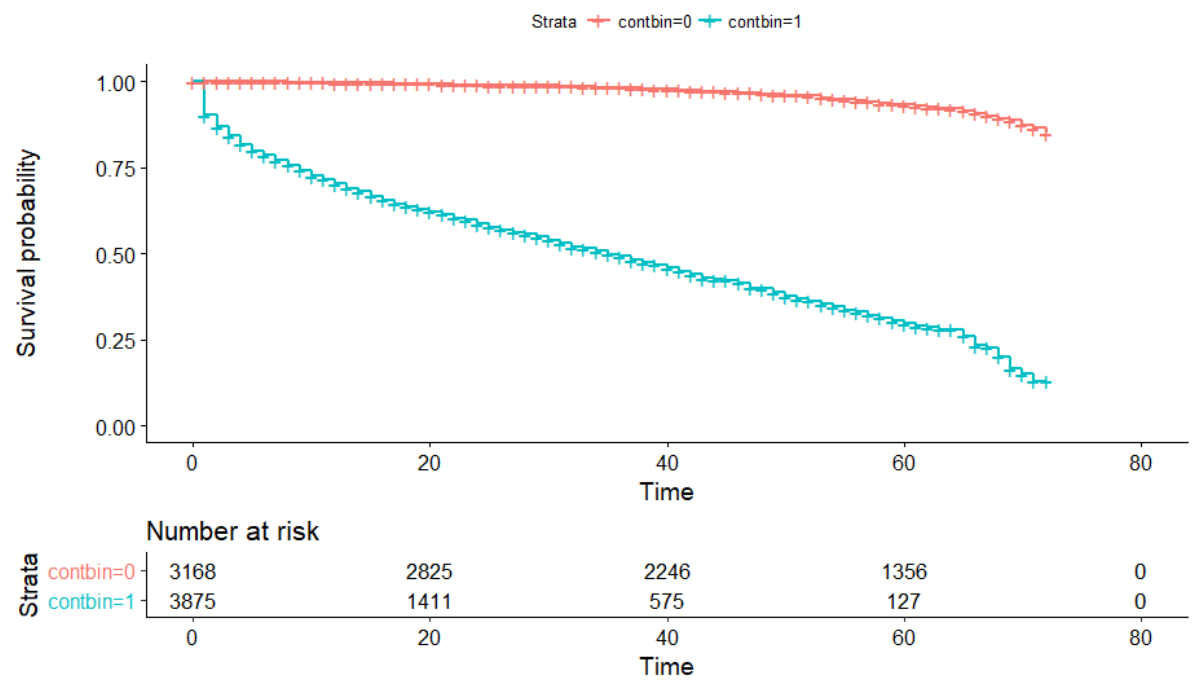

Here contbin $=\mathbf{0}$ represent the Postpaid Subscribers, while contbin=1 represents the Prepaid Subscribers; the time unit is months. It's apparent from the 
plot that the survival probability for Prepaid users is very much less than Postpaid's: whereas $90 \%$ of Postpaids survive for 70 months or more, only less than $15 \%$ of Prepaids survive for the same period. Furthermore, the rapid hazard onset for Prepaid is notorious, having only $60 \%$ of survival probability around the 20 month mark.

Now we take exclusively the population of Prepaid subscribers to analyze their behavior and try to fit a good model for predicting churn. When fitting a polynomial power regression curve to this data, we arrived at the following empirical curve:

Fig.3.Polynomial fit

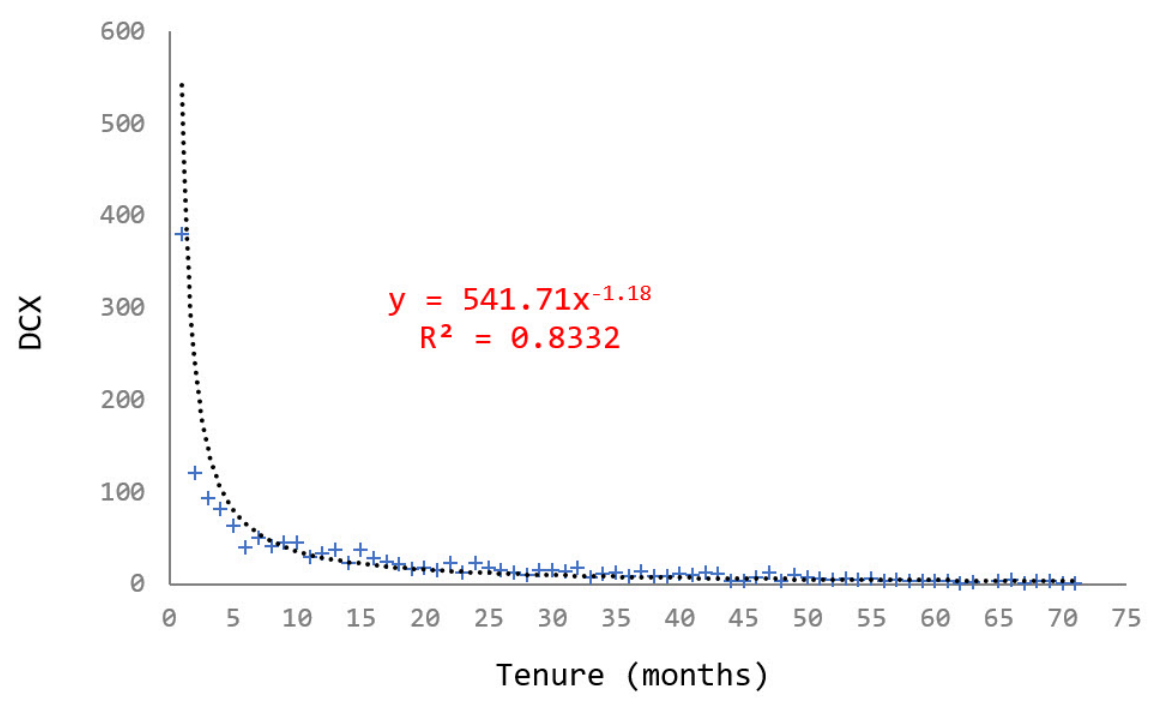

We obtain a .8332 value for $R^{2}$ which, while good, could be improved by the choice of a better distribution fit. The shape of the curve resembles a decreasing Weibull distribution, used in reliability engineering to estimate the probability of failure of components, gears and other manufactured items. Such a distribution is defined by two parameters $\lambda$ and $p$ with a probability density function of the form:

$$
f(t)=\lambda p t^{-1} e\left(-\lambda t^{p}\right)
$$

with $p$ being the shape parameter and $\lambda$ being the scale parameter. It follows from (7) that the hazard rate will:

- decrease over time if $p<1$

- remain constant if $p=1$

- increase over time if $p>0$ 
In our case, $p<1$ since the churn is diminishing over time. Next, we will proceed to apply a Weibull regression fit to the data to measure performance.

Our objectives are to minimize the error between the data and the empirical curve and to maximize the predictive power of the model. Generating the plot for the Weibull fit we arrive at the following curve

Fig.4.Weibull fit

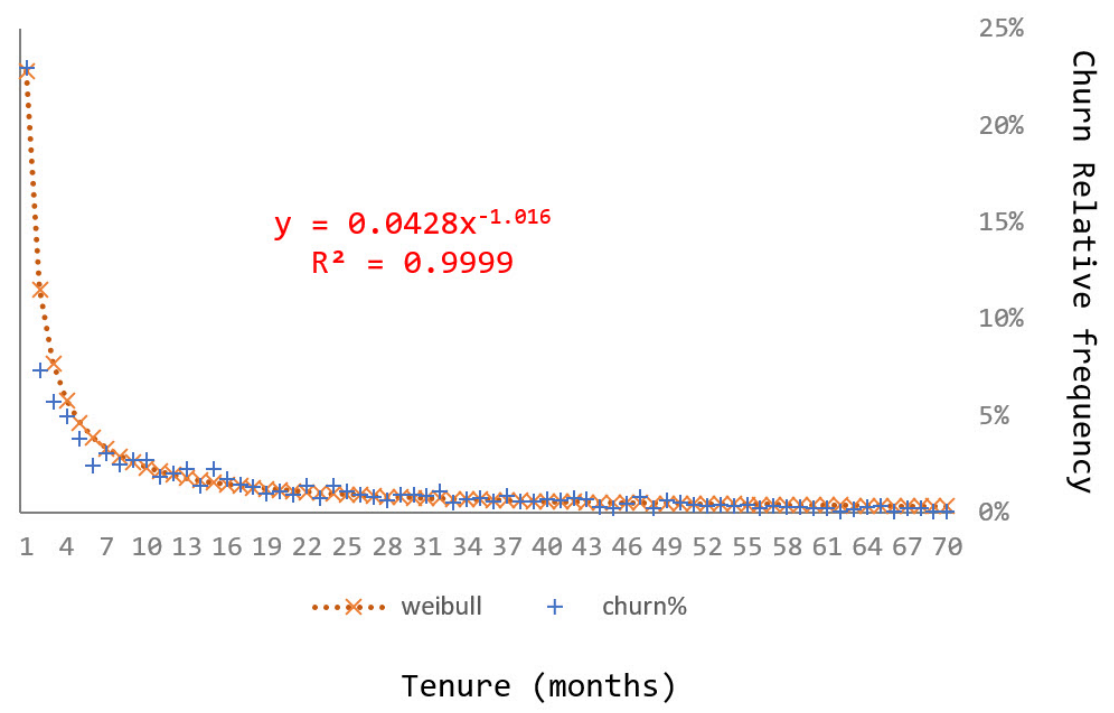

\section{$6 \quad$ Results and findings}

With a $R^{2}$ of .9999 , we've obtained a perfect fit for our model; however, to avoid the trap of falling in over-fitting, we also test for the $R$ predicted statistic, to actually assess the predictive power. $R_{\text {pred }}$ is a robust metric to measure the out-of-the-data set capability of the model to fit new data, rather than just fitting the sample set.

$R_{\text {pred }}$ is computed as follows:

$R_{\text {pred }}=1-\frac{P R E S S}{S S T O}$ 
where PRESS is the Prediction Sum of Squares given by

$$
\sum_{i=1}^{n}\left(y_{i}-\widehat{y}_{i(i)}\right)^{2}
$$

and SSTO is the Total Sum of Squares, obtained from

$$
\sum_{i=1}^{n}\left(y_{i}-\bar{y}\right)^{2}
$$

Our first attempt to model with a polynomial-power regression yielded a $R^{2}$ of. 8332 and a $R_{\text {pred }}$ of $74 \%$, meaning we could predict or forecast a future value of churn with $74 \%$ accuracy. The Weibull fit model, output a $.9999 R^{2}$ and a $R_{\text {pred }}$ of $99.85 \%$, showing not only an internal goodness of fit but also an optimal predictive power for future estimations out of the evaluated data set. Marketing-wise, this high value for $R_{\text {pred }}$ coincides with the company improving his customer relationship over time; also with the optimizing of product benefits and with the generation of brand love.

From the perspective of the customers, the model is coherent with them getting used to the service and developing a resistance to change or terminate the subscription to move on to another carrier. It is this balanced ecosystem which provides the best result in the long term for both, company and customer.

\section{Conclusions}

In this paper we have shown that traditional churn models with fixed deterministic variables are not good estimators of Prepaid churn. We showed that churn could be modeled as hazard function akin to failure rates in reliability engineering and applied a Weibull regression fit to estimate Prepaid churn behavior. This behavior shows rapid hazard onset at the beginning of the periods evaluated, but then normalizes and flattens out as time progresses. We also

demonstrated that the Weibull model is not only a good fit for the sample data, but also displays excellent predictive power for the kind of data analyzed.

All the calculations where performed using $\mathrm{R}$ libraries survival and survminer, with aid from JASP software for cross-validation of the regression statistics. The library ggplot2 was used to generate the graphics. 


\section{References}

[1] IBM Watson telco customer demo dataset. https://www.ibm.com/communities/analytics/watsonanalytics-blog/predictive-insights-in-the-telco-customer-churn-data-set/

[2] https://www.gsmaintelligence.com/dashboards

[3] Tallberg, M. Bundling of Handset and Subscription. Helsinki University of Technology (2004)

[4]Figini, S. Customer relationship: a survival analysis approach. Proceedings of COMPSTAT 2006, Roma, pp. 959-966

[5] Kaplan, E. L. and Meier, R. Nonparametric Estimation from Incomplete Observations. Journal of the American Statistical Association, 53, 457481, (1958).

[6] Mudholkar, G.S. and Srivastava, D.K. Exponentiated Weibull Family for Analyzing Bathtub Failure-Rate Data. IEEE Transactions on Reliability, Vol. 42. 1993.

[7] Zhang, Z. Parametric regression model for survival data: Weibull regression model as an example. Annals of Translational Medicine. v.4(24). 2016

[8] Leventhal, B. An Introduction to Survival Analysis. Henry Stewart Briefing on Marketing. Barry Analytics. 2010

[9] Dätwyler, C. and Stucki T. Parametric Survival Models. ETHZürich 2013. 